\title{
INCOMPLETE LAPLACE INTEGRALS: UNIFORM ASYMPTOTIC EXPANSION WITH APPLICATION TO THE INCOMPLETE BETA FUNCTION*
}

\author{
N. M. TEMME $\dagger$
}

Abstract. The incomplete Laplace integral

$$
\frac{1}{\Gamma(\lambda)} \int_{\alpha}^{\infty} t^{\lambda-1} e^{-z t} f(t) d t
$$

is considered for large values of $z$. Both $\lambda$ and $\alpha$ are uniformity parameters in $[0, \infty)$. The basic approximant is an incomplete gamma function, that is, the above integral with $f=1$. Also, a loop integral in the complex plane is considered with the same asymptotic features. The asymptotic expansions are furnished with error bounds for the remainders in the expansions. The results of the paper combine four kinds of asymptotic problems considered earlier. An application is given for the incomplete beta function. The present investigations are a continuation of earlier works of the author for the above integral with $\alpha=0$. The new results are significantly based on the previous case.

Key words. uniform asymptotic expansion of integrals, incomplete gamma function, incomplete beta function, incomplete Laplace integral, construction of error bounds

AMS(MOS) subject classification. $41 \mathrm{~A} 60,30 \mathrm{~F} 15,33 \mathrm{~A} 15,44 \mathrm{~A} 10$

1. Introduction. This paper is the third in a set dealing with uniform asymptotic expansions of Laplace type integrals. The previous papers are [11] and [12]. In the present paper, we consider the integral

$$
F_{\lambda}(z, \alpha)=\frac{1}{\Gamma(\lambda)} \int_{\alpha}^{\infty} t^{\lambda-1} e^{-z t} f(t) d t,
$$

where $z$ is a large parameter and $f$ is holomorphic in a domain $\Omega$ that contains the nonnegative reals; $\lambda, \alpha$ and $z$ are real variables for which the integral is properly defined. Say, $\alpha \geqq 0, \lambda \geqq 0$ and $z>0$. An interpretation of $F_{0}(z, 0)$ follows from

$$
\lim _{\lambda \rightarrow 0} F_{\lambda}(z, \alpha)= \begin{cases}0 & \text { if } \alpha>0, \\ f(0) & \text { if } \alpha=0 .\end{cases}
$$

The second case follows from integration by parts.

We are interested in the asymptotic expansion of (1.1) for $z \rightarrow \infty$ which is uniformly valid with respect to both $\lambda$ and $\alpha$ in $[0, \infty)$. The parameters $\lambda$ and $\alpha$ may be coupled with the large parameter $z$, or they may range independently through the uniformity interval. For a description of the various asymptotic features, four different cases with their own asymptotic phenomena can be distinguished.

(i) $\alpha$ fixed, $\lambda$ fixed. For this classical case Watson's lemma gives an expansion. When $\alpha=0, f(t)$ is expanded in powers of $t$, when $\alpha>0, t^{\lambda-1} f(t)$ is expanded in powers of $t-\alpha$. See [6, p. 113].

(ii) $\alpha \geqq 0, \lambda$ fixed. An incomplete gamma function (i.e., (1.1) with $f=1$ ) is needed to describe the uniform transition of $\alpha=0$ to $\alpha>0$; [4], [8], [9] and [14] are appropriate references. The asymptotic feature is the possible coalescence of two critical points: $t=0$ (an algebraic singularity) and $t=\alpha$ (end-point of integration).

\footnotetext{
* Received by the editors November 11, 1985; accepted for publication (in revised form) May 13, 1986.
}

$\dagger$ Centre for Mathematics and Computer Science, P.O. Box 4079, 1009 AB Amsterdam, The Netherlands. 
(iii) $\alpha=0, \lambda \geqq 0$. The saddle point of $t^{\lambda} e^{-z t}$, which occurs at $t=\mu:=\lambda / z$, may coalesce with $t=0$, the end-point of integration. In that event (i.e., when $\mu \rightarrow 0$ ) the saddle point disappears, since $e^{-z t}$ does not have a saddle point. No extra special function is needed to describe this feature; in fact the (complete) gamma function, which is incorporated in (1.1) for normalization, can handle this case. See [11] and [12].

(iv) $\alpha>0$ (fixed), $\lambda \geqq 0$. When $\mu:=\lambda / z$ is larger than $\alpha$, the saddle point is inside the interval of integration; otherwise it is outside. This transition can be described by using an error function. A transformation gives an integral of the form

$$
\int_{\eta}^{\infty} e^{-z u^{2}} g(u) d u,
$$

and here the transition occurs at $\eta=0$. See [10]; similar cases are considered in [2] and [13].

These four cases are combined in our approach, where $\alpha \geqq 0, \lambda \geqq 0$. As in case (ii), the basic approximant is the incomplete gamma function. However, in case (ii) the full ranges of both parameters of this approximant are not completely exploited. As discussed in [7], it is expected that a two-variable approximant is needed to handle a three-variable case as in (1.1).

Apart from combining four existing methods, our results are interesting in view of applications. We consider the well-known incomplete beta function $I_{x}(p, q)$, and we give an expansion for large values of $p$, valid uniformly with respect to both $x$ and $q ; x \in[0,1], q \geqq 0$. Since $I_{x}(p, q)=1-I_{1-x}(q, p)$, the parameters $p$ and $q$ are interchangeable. So we solve an open problem mentioned in [10], where the incomplete beta function is considered as belonging to case (ii), as well as to case (iv). A transition from one case to another was not available at that moment.

The plan of the paper is as follows. In $\S 2$ we give the formal expansion of (1.1). It appears that an essential part of the expansion is that of the complete integral (1.1) with $\alpha=0$. Subsequent sections give representations of the remainders, conditions on $f$, the asymptotic nature of the expansions and the construction of error bounds. In $\S 8$ we consider analogous results for loop integrals in the complex plane. A loop integral with essentially the same asymptotic features as (1.1) has the form

$$
\int t^{-\lambda} e^{z t} \frac{f(t)}{t-\alpha} d t
$$

For $\alpha=0$ it reduces, just as (1.1), to a form that we considered earlier. Section 9 gives two new expansions for the incomplete beta function

Terminology. We call a variable fixed when it is independent of $z, \lambda$ and $\alpha$. A sequence of functions $\left\{\psi_{s}\right\}$ is called an asymptotic scale when, for $s=0,1, \cdots, \psi_{s+1}=$ $o\left(\psi_{s}\right)$ as $z \rightarrow \infty$. The formal series $\sum_{s=0}^{\infty} f_{s}(z)$ is said to be an asymptotic expansion of $F(z)$ with respect to the scale $\left\{\psi_{s}\right\}$, if for $n=0,1,2, \cdots$

$$
F(z)-\sum_{s=0}^{n} f_{n}(z)=o\left(\psi_{n}\right) \quad \text { as } z \rightarrow \infty .
$$

In this case we write

$$
F(z) \sim \sum_{s=0}^{\infty} f_{s}(z), \quad\left\{\psi_{s}\right\} \quad \text { as } z \rightarrow \infty .
$$

In uniform expansions it is required that the " $o$ " symbols in (1.2) and in the definition of the scale hold uniformly (with respect to $\alpha, \lambda$ or $\mu=\lambda / z$ in certain domains, say). See [3]. 
2. Uniform expansions: construction of the formal series. Before considering the general case (1.1), we repeat the procedure for $F_{\lambda}(z, 0)$, which will be denoted by

$$
F_{\lambda}(z)=\frac{1}{\Gamma(\lambda)} \int_{0}^{\infty} t^{\lambda-1} e^{-z t} f(t) d t
$$

This function and its expansion play an essential role in the expansion of (1.1). The following integration by parts procedure takes into account the role of the critical point: the saddle point of $t^{\lambda} e^{-z t}$, i.e., the point $t=\mu$, where

$$
\mu=\lambda / z \text {. }
$$

We write

$$
f(t)=f(\mu)+(t-\mu) g(t)
$$

and we obtain

$$
\begin{aligned}
F_{\lambda}(z) & =f(\mu) z^{-\lambda}+\frac{1}{\Gamma(\lambda)} \int_{0}^{\infty} t^{\lambda-1} e^{-z t}(t-\mu) g(t) d t \\
& =f(\mu) z^{-\lambda}-\frac{1}{z \Gamma(\lambda)} \int_{0}^{\infty} g(t) d\left(t^{\lambda} e^{-z t}\right) \\
& =f(\mu) z^{-\lambda}+\frac{1}{z \Gamma(\lambda)} \int_{0}^{\infty} t^{\lambda-1} e^{-z t} f_{1}(t) d t
\end{aligned}
$$

where we assume that integrated terms at $t=0, t=\infty$ vanish, and

$$
f_{1}(t)=t \frac{d}{d t} g(t)=t \frac{d}{d t} \frac{f(t)-f(\mu)}{t-\mu} .
$$

Repeating this process, we obtain the formal expansion

$$
F_{\lambda}(z) \sim z^{-\lambda} \sum_{s=0}^{\infty} f_{s}(\mu) z^{-s}, \quad z \rightarrow \infty,
$$

where $f_{0}(t)=f(t)$ and

$$
f_{s+1}(t)=t \frac{d}{d t} \frac{f_{s}(t)-f_{s}(\mu)}{t-\mu}, \quad s=0,1, \cdots .
$$

For the general case (1.1) we again take (2.3) as the first step. Now we have an integrated term at $t=\alpha$. It is not difficult to see that we obtain the formal expansion

$$
F_{\lambda}(z, \alpha) \sim z^{-\lambda} Q(\lambda, \alpha z) \sum_{s=0}^{\infty} f_{s}(\mu) z^{-s}+\frac{\alpha^{\lambda} e^{-\alpha z}}{z \Gamma(\lambda)} \sum_{s=0}^{\infty} B_{s}(\alpha) z^{-s}, \quad z \rightarrow \infty,
$$

where $f_{s}(\mu)$ are the same as in $(2.4), B_{s}(\alpha)$ are defined by

$$
B_{s}(\alpha)=\frac{f_{s}(\alpha)-f_{s}(\mu)}{\alpha-\mu}, \quad s=0,1, \cdots,
$$

and $Q(a, x)$ is the incomplete gamma function ratio

$$
Q(a, x)=\frac{1}{\Gamma(\alpha)} \int_{x}^{\infty} t^{a-1} e^{-t} d t
$$

We observe that the first series in (2.6) does not depend on $\alpha$; in fact we recognize the expansion given in (2.4). Furthermore, the integrated terms at $t=\alpha$, which generate the second series, all vanish when $\alpha \rightarrow 0$. 
These observations lead us to the representation, including the definition of a new function $B_{\lambda}$,

$$
F_{\lambda}(z, \alpha)=Q(\lambda, \alpha z) F_{\lambda}(z)+\frac{\alpha^{\lambda} e^{-\alpha z}}{z \Gamma(\lambda)} B_{\lambda}(z, \alpha),
$$

which should not be interpreted as an asymptotic relation, but as an exact identity. We consider the incomplete gamma ratio as a known function, of which the asymptotic features are well known (see [10]). For numerical aspects concerning this function see [5]. As mentioned above, the asymptotic expansion of $F_{\lambda}(z)$, i.e., (2.4), is also settled earlier. More details on this point will be given below. So we are left with the function $B_{\lambda}(z, \alpha)$, of which the asymptotic expansion formally follows from the second series in (2.6).

A somewhat different method used to obtain the expansion for $B_{\lambda}(z, \alpha)$ is based on a differential equation for this function. By differentiating (2.9) with respect to $\alpha$, we easily obtain

$$
\frac{\alpha}{z} B_{\lambda}^{\prime}(z, \alpha)+(\mu-\alpha) B_{\lambda}(z, \alpha)=z^{\lambda} F_{\lambda}(z)-f(\alpha) .
$$

Substitution of (2.4) and of the formal series

$$
B_{\lambda}(z, \alpha) \sim \sum_{s=0}^{\infty} \frac{B_{s}(\alpha)}{z^{s}}
$$

into (2.10) shows that this equation is formally satisfied if

$$
\begin{aligned}
& (\mu-\alpha) B_{s}(\alpha)=f_{s}(\mu)-\alpha B_{s-1}^{\prime}(\alpha), \quad s=1,2, \cdots, \\
& B_{0}(\alpha)=[f(\alpha)-f(\mu)] /(\alpha-\mu) .
\end{aligned}
$$

Here, and in (2.10), the prime denotes differentiation with respect to $\alpha$. It easily follows that (2.12) generates the same coefficients $B_{s}(\alpha)$ as those defined in (2.7). Therefore, by using (2.9), we again arrive at (2.6).

The following integral

$$
E_{\lambda}(z, \alpha)=\frac{1}{\Gamma(\lambda)} \int_{0}^{\alpha} t^{\lambda-1} e^{-z t} f(t) d t
$$

is strongly related to (1.1). It has a similar representation as (2.9). When we use the following complementary relations

$$
E_{\lambda}(z, \alpha)+F_{\lambda}(z, \alpha)=F_{\lambda}(z), \quad Q(\lambda, \alpha z)+P(\lambda, \alpha z)=1,
$$

we obtain

$$
E_{\lambda}(z, \alpha)=P(\lambda, \alpha z) F_{\lambda}(z)-\frac{\alpha^{\lambda} e^{-\alpha z}}{z \Gamma(\lambda)} B_{\lambda}(z, \alpha) .
$$

Consequently, when we give expansions for $F_{\lambda}(a)$ and $B_{\lambda}(z, \alpha)$, the results can be used for both integrals (1.1) and (2.13). The function $P(a, x)$ again is an incomplete gamma function, with representation

$$
P(a, x)=\frac{1}{\Gamma(a)} \int_{0}^{x} t^{a-1} e^{-t} d t
$$


3. Representations for the remainders. We introduce remainders for (2.4) and (2.11) by writing

$$
\begin{aligned}
& z^{\lambda} F_{\lambda}(z)=\sum_{s=0}^{n-1} f_{s}(\mu) z^{-s}+z^{-n} \bar{f}_{n}, \\
& B_{\lambda}(z, \alpha)=\sum_{s=0}^{n-1} B_{s}(\alpha) z^{-s}+z^{-n} \bar{B}_{n},
\end{aligned}
$$

where $n=0,1,2, \cdots$. When $n=0$ the sums are empty and they have to be replaced by 0 .

The procedure leading to $(2.4)$ yields for $\bar{f}_{n}$ the representation

$$
\bar{f}_{n}=\frac{z^{\lambda}}{\Gamma(\lambda)} \int_{0}^{\infty} t^{\lambda-1} e^{-z t} f_{n}(t) d t .
$$

To obtain a representation for $\bar{B}_{n}$, we write (2.9) in the form

$$
B_{\lambda}(z, \alpha)=z e^{\alpha z} \alpha^{-\lambda} \int_{\alpha}^{\infty} t^{\lambda-1} e^{-z t}\left[f(t)-z^{\lambda} F_{\lambda}(z)\right] d t .
$$

Writing

$$
B_{\lambda}(z, \alpha)=B_{0}(\alpha)+z^{-1} \bar{B}_{1}, \quad z^{\lambda} F_{\lambda}(z)=f(\mu)+z^{-1} \bar{f}_{1},
$$

and using integration by parts in the form

$$
t^{\lambda-1} e^{-z t} d t=\frac{-1}{z(t-\mu)} d\left(t^{\lambda} e^{-z t}\right)
$$

we obtain

$$
\bar{B}_{1}=z \alpha^{-\lambda} e^{\alpha z} \int_{\alpha}^{\infty} t^{\lambda-1} e^{-z t}\left[f_{1}(t)-\bar{f}_{1}\right] d t .
$$

Repeating this, and using the recursions

$$
\bar{B}_{n}=B_{n}(\alpha)+z^{-1} \bar{B}_{n+1}, \quad \bar{f}_{n}=f_{n}(\mu)+z^{-1} \bar{f}_{n+1},
$$

we finally have

$$
\bar{B}_{n}=z \alpha^{-\lambda} e^{\alpha z} \int_{\alpha}^{\infty} t^{\lambda-1} e^{-z t}\left[f_{n}(t)-\bar{f}_{n}\right] d t,
$$

where $n=0,1,2, \cdots$. For $n=0$ this equals the starting point (3.4). An equivalent representation is

$$
\bar{B}_{n}=-z \alpha^{-\lambda} e^{\alpha z} \int_{0}^{\alpha} t^{\lambda-1} e^{-z t}\left[f_{n}(t)-\bar{f}_{n}\right] d t,
$$

which easily follows by writing $\int_{\alpha}^{\infty}=\int_{0}^{\infty}-\int_{0}^{\alpha}$ and using (3.3).

The availability of both forms (3.5) and (3.6) is important in the analysis to be given below. Namely, for bounding $\bar{B}_{n}$ we always have an integral in which the saddle point $\mu$ is not an interior point of the interval of integration.

The above representations for $\bar{f}_{n}$ and $\bar{B}_{n}$ are formally obtained. In the next section we give the conditions on $f$ to justify the above results, and to discuss the asymptotic nature of the expansions. 
4. Assumptions on $f$. We consider real values of $\alpha, \lambda$ and $z$, with $\alpha, \lambda \geqq 0, z>0$. We accept that $f$ depends on the uniformity parameter $\mu$ defined in (2.2). The reason is that in applications usually a transformation to the standard form is needed, which yields a function $f$ depending on $\mu$. In [12] a detailed discussion of such a transformation is given. By means of several examples, it is shown that the assumption $f$ depends on $\mu$ may be relevant and quite acceptable. The parameter $\alpha$ plays a completely different role, and we do not suppose that $f$ depends on it. The example in $\S 9$ on the incomplete beta function shows more details on the transformation to the standard form (1.1), and on the role of $\alpha$ and $\mu$.

The analysis is based on the assumption that $f$ is holomorphic in a domain of the complex plane. Again, for applications in the theory of special functions, this condition is quite natural. Another point is that part of the analysis runs rather elegantly when using complex function theory. However, the construction of the expansions, the representations of the remainders in (3.3), (3.5), and the construction of error bounds can also be given for functions $f$ belonging to continuity classes $C^{k}([0, \infty))$. When $k<\infty$ we cannot, of course, define the complete expansions (2.4), (2.11).

We assume that $f$ is holomorphic in a simply connected domain $\Omega$ of the complex plane; $\Omega$ may depend on $\mu$ and $\Omega$ should contain $\mathbb{R}^{+}$. We suppose that the distance $d(t)$ from $t \in \mathbb{R}^{+}$to the boundary $\partial \Omega$ of $\Omega$ is increasing according to the following requirement:

$$
d(t) \geqq d_{0}(\delta+t)^{\kappa}, \quad t \geqq 0 .
$$

where $\delta, d_{0}$ and $\kappa$ are fixed, $\delta, d_{0}>0$, and $\frac{1}{2} \leqq \kappa \leqq 1$.

It follows that, for large $\mu$, the singularities of $f$ are rather far from the saddle point $t=\mu$, this distance being $\mathcal{O}\left(\mu^{\kappa}\right)$. This condition is important for investigating the asymptotic nature of the expansion (2.4). The requirement that (4.1) holds for any positive $t$, and not only for $t=\mu$, is important for expansion (2.11).

A geometrical interpretation of (4.1) is as follows. Let $D_{t}$ be the disc around $t \in \mathbb{R}^{+}$ with radius $d_{0}(\delta+t)^{\kappa}$. Then the above condition implies that $\Omega$ contains the subset

$$
\Omega_{0}=\bigcup_{t \geqq 0} D_{t} .
$$

When $\kappa=\frac{1}{2}$ the boundary $\partial \Omega_{0}$ of $\Omega_{0}$ is a parabola; that is,

$$
\partial \Omega_{0}=\left\{t=u+i \nu \mid \nu^{2}=d_{0}^{2}\left(u+\delta+\frac{1}{4} d_{0}^{2}\right)\right\} .
$$

When $\kappa=1$ we have two possibilities depending on $d_{0}$ :

(i) $0<d_{0} \leqq 1, \Omega_{0}$ is a sector with vertex at $t=-\delta$ such that $|\arg (t+\delta)| \leqq$ $\arcsin \left(d_{0}\right)$; when $d_{0}=1$ this sector is the half-plane Re $t \geqq-\delta$.

(ii) $d_{0}>1, \Omega_{0}=\mathbb{C}$.

It is clear that for $\frac{1}{2}<\kappa<1$ the set $\Omega_{0}$ is something "between" a parabola-shaped domain and a sector. Geometrically, values of $\kappa$ larger than unity make no sense, although the analysis will accept such values.

We also need a growth condition on $f$ in $\Omega_{0}$. We assume that, when $\mu$ is fixed, $f$ is of algebraic growth at infinity. That is,

$$
M(\mu)=\sup _{t \in \Omega}\left(1+|t|^{-p}\right)|f(t)|
$$

should exist for all finite values of $\mu$ in $[0, \infty)$. Condition (4.4) will not exclude functions in (1.1) that can be written as

$$
f(t)=e^{\sigma t} \tilde{f}(t), \quad \sigma \text { fixed in } \mathbb{C} .
$$

When in such a decomposition $\tilde{f}$ meets the above conditions, we absorb the exponential 
part of this splitting into $\exp (-z t)$ of (1.1), just by a shift in the large parameter $z$. Afterwards, we proceed with $\tilde{f}$.

5. Estimates for $f_{s}(t), f_{s}(\mu)$ and $B_{s}(\alpha)$. The conditions on $f$ yield estimates for the functions $f_{s}(t)$ defined in (2.5) and for the coefficients $f_{s}(\mu)$. With the help of these estimates, the asymptotic scales for the expansions (3.1), (3.2) are chosen.

Let $\Omega_{r}$ be the subset of $\Omega_{0}$ defined by

$$
\Omega_{r}=\bigcup_{t \geqq 0} \tilde{D}_{t}
$$

where $\tilde{D}_{t}$ is a disc around $t$ with radius $r(\tilde{\delta}+t)^{\kappa}$, with $0<\tilde{\delta}<\delta, 0<r<d_{0}, \tilde{\delta}$ and $r$ fixed. Then the derivatives of $f$ at $t$ can be written as

$$
f^{(s)}(t)=\frac{s !}{2 \pi i} \int_{C_{r}} \frac{f(\tau)}{(\tau-t)^{s+1}} d \tau,
$$

where $C_{r}$ is the boundary of $\tilde{D}_{t}$.

It follows that we can assign numbers $K_{s}$, not depending on $t$ and $\mu$, such that

$$
\left|f^{(s)}(t)\right| \leqq K_{s} M(\mu)(1+|t|)^{p-s \kappa}, \quad s=0,1,2, \cdots,
$$

for all $t \in \Omega_{r}$, and all $\mu \in[0, \infty)$. That is,

$$
f^{(s)}(t)=M(\mu)(1+|t|)^{p-s \kappa} \mathscr{O}(1), \quad s=0,1,2, \cdots,
$$

with $t \in \Omega_{r}$, uniformly with respect to $\mu$ in $[0, \infty)$.

The functions $f_{s}(t)$ defined in (2.5) are analytic in $\Omega$. They can be expressed in terms of the derivatives of $f$, as follows from their definition. For $t$-values near $\mu$, the functions cannot be estimated by repeated application of (2.5), owing to the factors $1 /(t-\mu)$ and powers of it. Another approach is using the mean value theorem on

$$
\begin{aligned}
f_{s}(t) & =t \frac{d}{d t} \int_{0}^{1} f_{s-1}^{\prime}[\mu+\tau(t-\mu)] d \tau \\
& =t \int_{0}^{1} \tau f_{s-1}^{\prime \prime}[\mu+\tau(t-\mu)] d \tau,
\end{aligned}
$$

which gives $f_{s}(t)=\frac{1}{2} t f_{s-1}^{\prime \prime}\left(\tau_{s}\right)$, where $\tau_{s}$ is a value between $t$ and $\mu$. By repeating this, we obtain

$$
f_{s}(t)=t \sum_{j=0}^{s-1} p_{j} f^{(s+j+1)}\left(\tau_{j}\right), \quad s \geqq 1,
$$

where $\tau_{j}$ are between $t$ and $\mu$, and $p_{j}$ is a homogeneous polynomial of degree $j$ of $j$ variables, all between $t$ and $\mu$. The coefficients of $p_{j}$ do not depend on $\mu$ and $t$. Therefore, we have

$$
\begin{aligned}
& p_{j}=(1+\mu+t)^{j} \mathcal{O}(1), \\
& f^{(s+j+1)}\left(\tau_{j}\right)=M(\mu)(1+\mu+t)^{p-(s+j+1) \kappa} \mathcal{O}(1),
\end{aligned}
$$

with $\mu \geqq 0, t \geqq 0$. It follows that (5.4) can be written as

$$
f_{s}(t)=t M(\mu)(1+\mu+t)^{p-1-s \bar{\kappa}} \mathbb{O}(1), \quad \bar{\kappa}=2 \kappa-1, \quad s=1,2, \cdots,
$$

for all $t \in \Omega_{r}$, uniformly with respect to $\mu \in[0, \infty)$. 
The values $f_{s}(\mu)$, which are the coefficients of the expansion (2.4), can be written as

$$
f_{s}(\mu)=\mu \sum_{j=0}^{s-1} q_{j} \mu^{j} f^{(s+j+1)}(\mu), \quad s=1,2, \cdots,
$$

where $q_{j}$ are fixed numbers. An estimate as in (5.5) reads

$$
f_{s}(\mu)=\mu M(\mu)(1+\mu)^{p-1-s \bar{\kappa}} \mathcal{O}(1), \quad \bar{\kappa}=2 \kappa-1, \quad s=1,2, \cdots, \quad \mu \geqq 0 .
$$

The coefficients $B_{s}(\alpha)$ defined in (2.7) can be estimated by means of

$$
B_{s}(\alpha)=\int_{0}^{1} f_{s}^{\prime}[\mu+\tau(\alpha-\mu)] d \tau=f_{s}^{\prime}\left(\tau_{s}\right),
$$

where $\tau_{s}$ is between $\mu$ and $\alpha$. Therefore,

$$
B_{s}(\alpha)=\frac{1}{2 \pi_{i}} \int_{C_{r}} \frac{f_{s}^{\prime}(\tau)}{\left(\tau-\tau_{s}\right)^{2}} d \tau,
$$

where $C_{r}$ is a circle in $\Omega_{r}$ around $\tau_{s}$ with radius $\mathcal{O}\left(1+\tau_{s}\right)^{\kappa}=\mathcal{O}(1+\mu+\alpha)^{\kappa}$. By using (5.5), it follows that

$$
B_{s}(\alpha)=M(\mu)(1+\mu+\alpha)^{p-s \bar{\kappa}-\kappa} \mathcal{O}(1), \quad s=0,1, \cdots,
$$

with $\alpha, \mu \geqq 0$.

Example 5.1. When $f(t)=1 /(1+t)$, we have $p=\kappa=1$, and $M$ slightly larger than 1. The first coefficients $B_{0}, B_{1}$ are

$$
B_{0}(\alpha)=-1 /[(\alpha+1)(\mu+1)], \quad B_{1}(\alpha)=(1-\alpha \mu) /\left[(\alpha+1)^{2}(\mu+1)^{3}\right],
$$

which confirms (5.7).

To conclude this section we consider limiting values of $\bar{B}_{n}$ at $\alpha=0$ and $\alpha=\infty$. For $\alpha \rightarrow 0$ we write $t=\alpha \tau$ in (3.6). We obtain

$$
\bar{B}_{n}=\frac{\bar{f}_{n}-f_{n}(0)}{\mu} \text { at } \alpha=0 .
$$

This expression is regular at $\mu=0$. To see this, replace (5.8) by

$$
\bar{B}_{n}=\frac{f_{n}(\mu)-f_{n}(0)}{\mu}+\frac{1}{\lambda} \bar{f}_{n+1} .
$$

From (2.5) we see that $f_{n}(t) / t$ is regular at $t=0$, when $n \geqq 1$. Hence, using (3.3), we obtain

$$
\bar{B}_{n}=f_{n}^{\prime}(0)+\int_{0}^{\infty} e^{-z t} f_{n+1}(t) t^{-1} d t \quad \text { at } \alpha=0, \mu=0, \text { and } n=0,1, \cdots
$$

For the limiting value of $\bar{B}_{n}$ at $\alpha=\infty$, consider (3.5) in the following form:

$$
\bar{B}_{n}=z \int_{0}^{\infty}(1+t)^{\lambda-1} e^{-\alpha z t}\left[f_{n}(\alpha(1+t))-\bar{f}_{n}\right] d t .
$$

Using (5.5), and considering $\alpha \gg \mu$, we can easily estimate $\bar{B}_{n}$ as $\alpha \rightarrow \infty$. For instance, when $p-n \bar{\kappa}=1$ and $\lim _{t \rightarrow \infty} f_{n}(t) / t$ exists (and is $L$ ), we have

$$
\bar{B}_{n}=L \quad \text { at } \alpha=\infty, \quad \mu \text { finite. }
$$


6. The asymptotic nature of the expansions. First we discuss the expansions (2.4), (3.1), although the complete integral (2.1) is considered in the previous paper [12]. However, there we mainly investigated an expansion somewhat different from (2.4) and, therefore, it is appropriate to consider (2.4), (3.1) in the present set-up once again. writing

6.1. The asymptotic nature of (3.1). We introduce the asymptotic scale $\left\{\psi_{s} z^{-s}\right\}$ by

$$
\begin{aligned}
& \psi_{0}=M(\mu)(1+\mu)^{p}, \\
& \psi_{s}=\mu M(\mu)(1+\mu)^{p-1-s \bar{\kappa}}, \quad s=1,2, \cdots,
\end{aligned}
$$

which is suggested by (5.6). Since we allow $f$ to depend on $\mu$, we have to use a scale that reflects the possible growth of $f$ when $\mu$ ranges in the domain $[0, \infty)$. With the above scale we are able to control the behaviour of the remainder $\bar{f}_{n}$ defined in (3.3).

It is easily verified that $\left\{\psi_{s} z^{-s}\right\}$ is a uniform asymptotic scale with $z$ as large parameter and $\mu$ as uniformity parameter in $[0, \infty)$. Moreover, when $\kappa>\frac{1}{2}$ (i.e., $\bar{\kappa}>0$ ) it also is an asymptotic scale for $\mu \rightarrow \infty$, uniformity with respect to $z \in\left[z_{0}, \infty\right), z_{0}$ being a fixed positive number. Observe that for $\kappa<\frac{1}{2}$ the scale fails to be uniform with respect to $\mu$ on $[0, \infty)$, but it still is on compact subsets of $[0, \infty)$.

THEOREM 6.1. For the expansions (2.4), (3.1) we can write

$$
z^{\lambda} F_{\lambda}(z) \sim \sum_{s=0}^{\infty} f_{s}(\mu) z^{-s}, \quad\left\{\psi_{s} z^{-s}\right\} \quad \text { as } z \rightarrow \infty,
$$

uniformly with respect to $\mu=\lambda / z$ in $[0, \infty)$.

Proof. It is sufficient to show that $\bar{f}_{n}=\mathcal{O}\left(\psi_{n}\right)$, where $\bar{f}_{n}$ is defined in (3.3). The interval of integration in (3.3) is split up as follows:

$$
[0, \infty)=\Delta_{-} \cup\left[t_{-}, t_{+}\right] \cup \Delta_{+},
$$

where

$$
\Delta_{-}=\left[0, t_{-}\right], \quad \Delta_{+}=\left[t_{+}, \infty\right), \quad t_{ \pm}=\mu \pm \varepsilon(\mu+1)^{\kappa},
$$

with $\varepsilon$ fixed, and small enough such that $\left[t_{-}, t_{+}\right]$lies inside $\Omega_{r}$ of $(5.1)$. When $t_{-}$ happens to be negative, we replace it by 0 . For $t \in\left[t_{-}, t_{+}\right]$we have $t=\mathcal{O}(\mu)$. Therefore, (5.5) yields

$$
f_{s}(t)=\mu M(\mu)(1+\mu)^{p-1-s \bar{\kappa}} \mathcal{O}(1), \quad s=1,2, \cdots .
$$

Hence, (3.3) can be written as

$$
\bar{f}_{n}=I_{-}+I_{+}+\mathcal{O}\left(\psi_{n}\right) \text { as } z \rightarrow \infty,
$$

where $I_{ \pm}$are the contributions to (3.3) from $\Delta_{ \pm}$. They are of order $\mathcal{O}\left(\psi_{n}\right)$, also. It is possible to show more: they are asymptotically equal to 0 with respect to the scale $\left\{\psi_{s}\right\}$. That is, $I_{ \pm}=\mathcal{O}\left(\psi_{m}\right)$ for any $m$ as $z \rightarrow \infty$, uniformly with respect to $\mu \in[0, \infty)$.

Again, the proof can be based on the estimates given in (5.5). In [12, § 3.4] a detailed analysis is given for proving that contributions from $\Delta_{ \pm}$for similar integrals are asymptotically negligible. This analysis will not be repeated here.

6.2. Two lemmas for (3.2). The next step is to consider (3.2), and to estimate the remainder defined in (3.5), (3.6). The analysis boils down to the following two lemmas, the results of which are formulated in terms of strict inequalities. So we are able to use them once again in $\S 7$ for deriving strict error bounds. 


\section{LemMa 6.1. Consider the function}

$$
g(\alpha, \lambda, z)=\alpha^{-\lambda} e^{\alpha z} \int_{0}^{\alpha} t^{\lambda} e^{-z t} d t,
$$

where $0 \leqq \alpha \leqq \mu, \mu=\lambda / z$. Let $\zeta$ be defined by $\frac{1}{2} \zeta^{2}=\alpha-\mu+\mu \log (\mu / \alpha), \zeta \geqq 0$. Then for $z>0$

$$
g(\alpha, \lambda, z) \leqq \min \left\{\frac{\alpha}{(\mu-\alpha) z}, \frac{\alpha \zeta}{\mu-\alpha} \sqrt{\frac{\pi}{2 \zeta}}\right\} .
$$

Proof. Write $g$ in the form

$$
g(\alpha, \lambda, z)=\int_{0}^{\alpha} e^{-z \phi(t)} d t, \quad \phi(t)=t-\mu \log t-\alpha+\mu \log \alpha .
$$

Integrating with respect to $\phi$, we have for $0 \leqq \alpha<\mu$

$$
g(\alpha, \lambda, z)=\int_{0}^{\infty} e^{-z \phi} \frac{t}{\mu-t} d \phi \leqq \frac{\alpha}{(\mu-\alpha) z},
$$

which gives the first possibility in (6.6). To obtain the second one we write

$$
\phi(t)=\frac{1}{2} w^{2}+\zeta w, \quad w \geqq-\zeta,
$$

with the corresponding relations

$$
t=0 \leftrightarrow w=+\infty, \quad t=\alpha \leftrightarrow w=0, \quad t=\mu \leftrightarrow w=-\zeta,
$$

where $\zeta$ is defined above. Now we obtain

$$
g(\alpha, \lambda, z)=\int_{0}^{\infty} e^{-z\left[w^{2} / 2+\zeta w\right]} f(w) d w, \quad f(w)=\frac{t(w+\zeta)}{\mu-t} .
$$

We have $0 \leqq f(w) \leqq f(0), w \geqq 0$. To verify the upper bound, we write

$$
f^{2}(w)=2 t \frac{1-x+x \log x}{(x-1)^{2}}, \quad x=\frac{\mu}{t} .
$$

The $x$-part of this is monotonically decreasing on the $x$-interval $[1, \infty) ; x=\mu / \alpha$ corresponds to $w=0$. Hence we obtain $f(w) \leqq \alpha \zeta /(\mu-\alpha)$. It follows that

$$
g(\alpha, \lambda, z) \leqq \frac{\alpha \zeta}{\mu-\alpha} \int_{0}^{\infty} e^{-z w^{2} / 2} d w
$$

which gives the second possibility in (6.6). This proves the lemma.

LEMMA 6.2. Consider the function

$$
G_{q}(\alpha, \lambda, z)=\alpha^{-\lambda} e^{\alpha z}(1+\alpha)^{-q} \int_{\alpha}^{\infty} t^{\lambda-1} e^{-z t}(1+t)^{q} d t
$$

where $\alpha \geqq 0, \mu \leqq \alpha, \mu=\lambda / z, q$ fixed, $q \in \mathbb{R}$. Let $\zeta$ be defined by

$$
\zeta= \begin{cases}+\infty, & \mu<0, \\ +\left[2\left(\alpha-\mu+\mu \log \frac{\mu}{\alpha}\right)\right]^{1 / 2}, & 0 \leqq \mu \leqq \alpha .\end{cases}
$$

Then for $z>0$

$$
G_{0}(\alpha, \lambda, z) \leqq \min \left\{\frac{1}{(\alpha-\mu) z}, \frac{\zeta}{\alpha-\mu} \sqrt{\frac{\pi}{2 z}}\right\}
$$


Furthermore, when $0 \leqq \mu \leqq \alpha$,

$$
G_{q}(\alpha, \lambda, z)=\mathcal{O}\left[G_{0}(\alpha, \lambda, z)\right] \text { as } z \rightarrow \infty,
$$

uniformly with respect to $\alpha, \mu$.

Proof. We first consider $G_{0}$, for which we obtain

$$
G_{0}(\alpha, \lambda, z)=\int_{\alpha}^{\infty} e^{-z \phi(t)} \frac{d t}{t}=\int_{0}^{\infty} e^{-z \phi} \frac{d \phi}{t-\mu} \leqq \frac{1}{(\alpha-\mu) z},
$$

where $\phi(t)$ is the same as in Lemma 6.1. Observe that this result also holds for negative values of $\mu$. For the second possibility in (6.8), we proceed with $\mu \geqq 0$. We again write $\frac{1}{2} w^{2}+\zeta w=\phi(t), w \geqq-\zeta$, now with the correspondences

$$
t=\infty \leftrightarrow w=\infty, \quad t=\alpha \leftrightarrow w=0, \quad t=\mu \leftrightarrow w=-\zeta .
$$

It follows that

$$
G_{0}(\alpha, \lambda, z)=\int_{0}^{\infty} e^{-z\left[w^{2} / 2+\zeta w\right]} f(w) d w
$$

with $f(w)=(w+\zeta) /(t-\mu)$. Using

$$
f^{2}(w)=\frac{2}{\mu} \frac{x-\log x-1}{(x-1)^{2}} \leqq f^{2}(0), \quad x=\frac{t}{\mu} \geqq 1,
$$

we obtain the second choice in (6.8).

When $q \leqq 0$ the proposition (6.9) is trivial. Writing

$$
G_{q+1}=\frac{1+\mu}{1+\alpha} G_{q}+\frac{\alpha^{-\lambda} e^{\alpha z}(1+\alpha)^{-q-1}}{-z} \int_{\alpha}^{\infty}(1+t)^{q} d\left(e^{-z t} t^{\lambda}\right),
$$

we obtain by performing integration by parts

$$
G_{q+1}=\frac{1+\mu}{1+\alpha} G_{q}+\frac{1}{z(1+\alpha)}-\frac{q}{z(1+\alpha)^{2}} G_{q-1}+\frac{q}{z(1+\alpha)} G_{q} .
$$

Since $q$ is fixed and $0 \leqq \mu \leqq \alpha$, the result (6.9) follows by recursion, say from negative $q$-values.

Remark 6.1. The first alternatives in both (6.6) and (6.8) grow indefinitely when $\alpha \rightarrow \mu$, whereas the other ones remain finite. We have

$$
\zeta /(\mu-\alpha) \rightarrow 1, \quad \zeta /(\alpha-\mu) \rightarrow 1,
$$

for (6.6), (6.8), respectively. Therefore, the second alternatives give a bound for $g$ and $G_{0}$ valid for the whole range of parameters given in the lemmas. The first bound is given since it is sharp when $z$ is large and $\alpha$ and $\mu$ are bounded away from each other. A more uniform description, which includes both alternatives in (6.6), (6.8), is possible, by using a bound in terms of an error function. That is, in fact, $g$ and $G_{0}$ can be estimated by

$$
f(0) \int_{0}^{\infty} e^{-z\left[w^{2} / 2+\zeta w\right]} d w=f(0) \sqrt{\frac{\pi}{2 z}} e^{z \zeta^{2} / 2} \operatorname{erf} c(\zeta \sqrt{z / 2}) .
$$

We take $\zeta=0$ because it gives a very simple and manageable result.

6.3. The asymptotic nature of (3.2). We proceed with (3.2), and we estimate the remainder $\bar{B}_{n}$ defined in (3.5), (3.6). We use the asymptotic scale $\left\{\chi_{s} z^{-s}\right\}$ defined by

$$
\chi_{s}=M(\mu)(1+\mu+\alpha)^{p-s \bar{\kappa}-\kappa}, \quad s=0,1,2, \cdots,
$$


$z$ is the large parameter, $\alpha$ and $\mu$ are uniformity parameters. The choice of scale is suggested by (5.7).

THEOREM 6.2. For the expansions (2.11) and (3.2) we can write

$$
B_{\lambda}(z, \alpha) \sim \sum_{s=0}^{\infty} B_{s}(\alpha) z^{-s}, \quad\left\{\chi_{s} z^{-s}\right\} \quad \text { as } z \rightarrow \infty,
$$

uniformly with respect to $\mu, \alpha$ in $[0, \infty)$.

Proof. All $\mathcal{O}$-symbols in the proof hold uniformly with respect to $\mu$ and $\alpha$ in $[0, \infty)$; the large parameter is not needed in some results.

It is sufficient to show that $\bar{B}_{n}$ of (3.5) or (3.6) is $\mathcal{O}\left(\chi_{n}\right)$. Write

$$
\bar{B}_{n}=B_{n}(\alpha)+z^{-1} \bar{B}_{n+1}, \quad n=0,1,2, \cdots .
$$

Since $B_{n}(\alpha)=\mathcal{O}\left(\chi_{n}\right)$, we proceed with $\bar{B}_{n+1}$. That is, we consider (3.5), (3.6) for $n \geqq 1$. We have two cases.

(i) $0 \leqq \alpha \leqq \mu$. In (3.6) we use

$$
\begin{aligned}
& \bar{f}_{n}=\mathcal{O}\left(\psi_{n}\right)=\mu M(\mu)(1+\mu+\alpha)^{p-1-n \bar{\kappa}} \mathcal{O}(1), \\
& f_{n}(t)=t M(\mu)(1+\mu+\alpha)^{p-1-n \bar{\kappa}} \mathcal{O}(1),
\end{aligned}
$$

where the first line follows from Theorem 6.1 and the second one from (5.5). The estimate for $f_{n}(t)$ gives in (3.6) a contribution

$$
z M(\mu)(1+\mu+\alpha)^{p-1-n \bar{\kappa}} g(\alpha, \lambda, z) \mathcal{O}(1),
$$

where $g$ is defined in (6.5). The above estimate for $\bar{f}_{n}$ gives in (3.6) a contribution

$$
\begin{aligned}
z \mu M(\mu) & (1+\mu+\alpha)^{p-1-n \bar{\kappa}} \alpha^{-\lambda} e^{\alpha z} \mathcal{O}(1) \int_{0}^{\alpha} t^{\lambda-1} e^{-z t} d t \\
= & M(\mu)(1+\mu+\alpha)^{p-1-n \bar{\kappa}} \alpha^{-\lambda} e^{\alpha z} \mathcal{O}(1) \int_{0}^{\alpha} e^{-z t} d t^{\lambda} \\
= & z M(\mu)(1+\mu+\alpha)^{p-1-n \bar{\kappa}}\left[g(\alpha, \lambda, z) \mathcal{O}(1)+\mathcal{O}\left(z^{-1}\right)\right] .
\end{aligned}
$$

From Lemma 6.1 it follows that

$$
g(\alpha, \lambda, z)=\mathscr{O}(\sqrt{\alpha / z})=\mathscr{O}\left[(1+\mu+\alpha)^{\kappa} / \sqrt{z}\right] .
$$

Neglecting the term $\mathcal{O}\left(z^{-1}\right)$ in the last line of (6.14), we conclude that both (6.13) and (6.14) are estimated by

$$
\sqrt{z} M(\mu)(1+\mu+\alpha)^{p-1-n \bar{\kappa}+\kappa} \mathcal{O}(1) .
$$

Taking into account that in (6.12) $z^{-1} \bar{B}_{n+1}$ has to be considered, we obtain

$$
\bar{B}_{n}=\mathcal{O}\left(\chi_{n}\right)+M(\mu)(1+\mu+\alpha)^{p-1-(n+1) \bar{\kappa}+\kappa} \mathcal{O}\left(z^{-1 / 2}\right)=\mathcal{O}\left(\chi_{n}\right) .
$$

This finishes the first part of the proof.

(ii) $0 \leqq \mu \leqq \alpha$. In this case the starting point for $\bar{B}_{n}$ is (3.5). For $\bar{f}_{n}$ we take the representation as in the previous case, for $f_{n}(t)$ we consider (5.5). We integrate by parts in the contribution from $\bar{f}_{n}$. Starting with (6.12) and using Lemma 6.2 twice, with $q=0$ and with $q=p-1-(n+1) \bar{\kappa}$, we obtain the required estimate $\bar{B}_{n}=\mathcal{O}\left(\chi_{n}\right)$.

As remarked after the introduction of the scale functions $\psi_{s}$ in (6.1), the large parameter $z$ and the uniformity parameter $\mu$ are interchangeable, only if $\kappa>\frac{1}{2}$. It is important enough to formulate this property as a theorem. 
THEOREM 6.3. Let $\frac{1}{2}<\kappa \leqq 1$. Then in (6.2) $\mu$ may act as the large parameter and $z$ as the uniformity parameter. In (6.9), $\alpha$ (or $\mu$ ) may act as large parameter, $\mu$ (or $\alpha$ ) and $z$ as uniformity parameters. The uniformity domain for $\mu$ and $\alpha$ is $[0, \infty)$, for $z$ it is $\left[z_{0}, \infty\right), z_{0}$ being a fixed positive number.

Proof. The proof follows easily from the properties of the asymptotic scales used in (6.2) and (6.11), and from the proofs of the earlier theorems.

Remark 6.2. The above theorems are formulated and proved for real values of the parameters. By slight adaptation of the scales they hold for complex values of $\alpha$ and $\mu$, as long as these values are restricted to $\Omega_{r}$ introduced in (5.1). Some care in the interpretation of (1.1) is needed when $\alpha$ assumes complex values around the origin, since $t^{\lambda}$ is not single valued. However, the many-valuedness of $F_{\lambda}(z, \alpha)$ is completely described in (2.9) by the known functions $Q(\lambda, \alpha z)$ and $\alpha^{\lambda}$. From (3.6) it easily follows that $B_{\lambda}(z, \alpha)=\bar{B}_{0}$ is regular at $\alpha=0$, and in fact in $\Omega_{r}$. See also (5.8). Another problem is: How do we handle complex values of $z$ ? The holomorphic function $f$ in (1.1) allows the contour of integration to be deformed. When doing so, we can extend the domains for the parameters $z, \alpha$ and $\mu$ considerably. We will not go into further details here for this complicated technical problem.

Remark 6.3. It is tempting to take $\left\{f_{s}(\mu) z^{-s}\right\}$ and $\left\{B_{s}(\alpha) z^{-s}\right\}$ as asymptotic scales in (6.2) and (6.1.1). However, the conditions on $f$ do not imply that they have this property. When they do not the theorems may still be applicable; however, rather useless expansions may arise. It is instructive to consider what is happening in the case $f(t)=1+\exp (-t)$.

7. Error bounds for the remainders. The theorems of the previous section are based on the concept of generalized asymptotic expansions. The estimates for proving the asymptotic properties are given in terms of $\mathfrak{O}$-symbols. So far, no information is available on the sharpness of these estimates, say in terms of exact error bounds. That is, it would be interesting to have available an estimate in the form

$$
\left|\bar{f}_{n}\right| \leqq K_{n}\left|f_{n}(\mu)\right|, \quad \mu \geqq 0, \quad z \geqq z_{0}>0,
$$

instead of $\bar{f}_{n}=\mathcal{O}\left(\psi_{n}\right)$, used in Theorem 6.1. When $f_{n}(\mu)$ happens to vanish, (7.1) can be modified. $K_{n}$ in (7.1) may depend on $z$ and $\mu$.

The required form of the bound (7.1) reflects the expectation that $\bar{f}_{n}$ will not deviate too much from $f_{n}(\mu)$. For slowly varying functions $f$, say for $f(t)=1 /(1+t)$, this surely will be true, especially when $z$ is large. However, the scale functions $\psi_{n}, \chi_{n}$ are constructed in terms of the global estimate $M(\mu)$, introduced in (4.4). Consequently, the asymptotic scales used in the theorems may be too rough to describe what is really happening in the asymptotic expansions.

To show this by way of a simple example, we consider $f(t)=\exp [\mu /(1+t)]$. It is easily verified that is satisfies the conditions of $\S 4 ; \Omega=\mathbb{C} \backslash\{-1\}, \Omega_{0}$ is the half-plane $\operatorname{Re} t \geqq-\delta$, where $0<\delta<1$. In (4.4), $p=0$ and $M(\mu)=\exp [\mu /(1-\delta)]$, which is exponentially large, when $\mu$ is large. However, we expect that the remainder $\bar{f}_{n}$ in the expansion (3.1) is comparable with $f_{n}(\mu)$, which is only algebraic in $\mu$. Therefore, the theorems of the previous section are applicable, but the chosen asymptotic scales are not able to control the remainders of the expansion in a realistic way. This is especially true for expansion (6.2); for (6.9), which is more global in character due to the second uniformity parameter, the chosen scale may be more suitable. We want to emphasize that in this example only the scales default, whereas the expansions themselves are appropriate and may be of interest. The above noticed imperfections (see also Remark 6.3) are inherent in the definition of generalized asymptotic expansions. We have chosen this framework in order to be able to describe precisely the propositions and 
what we want to prove. We consider this important in asymptotics, especially when one or more uniformity parameters are involved. On the other hand, we have the need of constructing sharp error bounds for the remainders in the expansions, so that we can interpret the expansions in a realistic way. An ideal procedure would be a combination of both approaches, but in this stage, for lack of a unified approach, we prefer to discuss them separately.

7.1. Error bounds for (3.1). As remarked earlier, the quantities $f_{s}(\mu)$ and $B_{s}(\alpha)$ may be grossly overestimated by the global upper bound $M(\mu)$ introduced in (4.4). A better approach, say for arriving at (7.1), seems to be to give a sharp estimate for $f_{n}(t)$ near $t=\mu$, whereas the estimate "far away" of this saddle point may be rather crude. To be more explicit, we need a comparison function $w(t, \mu), w: \mathbb{R}^{+} \times \mathbb{R}^{+} \rightarrow[1, \infty)$, that satisfies the condition $w(\mu, \mu)=1$, and that may be large outside an interval around $t=\mu$. We suppose that we can assign quantities $M_{n}$, which may depend on $\mu$ and which are strictly larger than unity:

$$
M_{n} \geqq 1+\varepsilon_{n}, \quad \varepsilon_{n} \text { fixed and positive, }
$$

such that for all $t \geqq 0$ we have

$$
\left|f_{n}(t)\right| \leqq M_{n}\left|f_{n}(\mu)\right| w(t, \mu) .
$$

Furthermore, we suppose that it is easy to calculate or estimate the integral

$$
\left|\bar{f}_{n}\right| \leqq \frac{M_{n}\left|f_{n}(\mu)\right| z^{\lambda}}{\Gamma(\lambda)} \int_{0}^{\infty} t^{\lambda-1} e^{-z t} w(t, \mu) d t,
$$

obtained from (3.3) by bounding $f_{n}(t)$ in this way. When $f_{n}(\mu)$ happens to have zeros on $(0, \infty),(7.3)$ and (7.4) have to be modified, say by replacing $\left|f_{n}(\mu)\right|$ by $\delta_{n}+\left|f_{n}(\mu)\right|$, $\delta_{n}>0$.

Since $f$, and hence all $f_{n}$, have algebraic growth on $[0, \infty)$ (see (4.4)), it will be sufficient that $w(t, \mu)=\mathscr{O}[\exp (\sigma t)]$ for some positive $\sigma$. This suggests as a possible choice $w(t, \mu)=\cosh [\sigma(t-\mu)]$, which meets all requirements formulated thus far. Substituting this into (7.4), we obtain

$$
\begin{aligned}
\frac{z^{\lambda}}{\Gamma(\lambda)} \int_{0}^{\infty} t^{\lambda-1} e^{-z t} \cosh [\sigma(t-\mu)] d t & =\frac{1}{2}\left[(1-\sigma / z)^{\lambda} e^{-\sigma \mu}+(1+\sigma / z)^{\lambda} e^{\sigma \mu}\right] \\
& =\mathcal{O}\left[\cosh \left(\frac{1}{2} \lambda \sigma^{2} / z^{2}\right)\right],
\end{aligned}
$$

as $z \rightarrow \infty$. When $\sigma=\mathcal{O}(1)(\mu \geqq 0)$, this contribution to the right-hand side of (7.4) is quite acceptable, so long as $\lambda=o\left(z^{2}\right)$. But for a uniformity domain $[0, \infty)$ it is unacceptable.

This brings us to a further requirement that

$$
\frac{z^{\lambda}}{\Gamma(\lambda)} \int_{0}^{\infty} t^{\lambda-1} e^{-z t} w(t, \mu) d t=\mathscr{O}(1),
$$

as $z \rightarrow \infty$, uniformly with respect to $\mu \in[0, \infty)$.

For several reasons the following comparison function is very convenient:

$$
w_{\sigma}(t, \mu)=\left[(t / \mu)^{-\mu} e^{t-\mu}\right]^{\sigma}, \quad \sigma \geqq 0,
$$

where $\sigma$ may depend on $\mu$, but not on $t$. For $\mu=0$ we define $w_{\sigma}(t, 0)=\exp (\sigma t)$. This choice fits better in the dominant part $t^{\lambda} e^{-z t}$ of (7.4) than the cosh-function tried before.

For (7.3) we write

$$
\left|f_{n}(t)\right| \leqq M_{n}\left|f_{n}(\mu)\right| w_{\sigma_{n}}(t, \mu)
$$


and (7.4) becomes

$$
\left|\bar{f}_{n}\right| \leqq M_{n} Q_{n}\left|f_{n}(\mu)\right|, \quad Q_{n}=\left(1-\sigma_{n} / z\right)^{-1 / 2} \Gamma^{*}\left(\lambda-\mu \sigma_{n}\right) / \Gamma^{*}(\lambda),
$$

where

$$
\Gamma^{*}(z)=[z /(2 \pi)]^{1 / 2} e^{z} z^{-z} \Gamma(z)=1+\mathcal{O}\left(z^{-1}\right),
$$

as $z \rightarrow \infty$. Compared with (7.5), this result is much more acceptable, since now we have

$$
Q_{n}=1+\mathcal{O}\left(z^{-1}\right) \text { as } z \rightarrow \infty,
$$

uniformly with respect to $\lambda$ or $\mu$ in $[0, \infty)$. Especially, large values of $\lambda$ are in favor in (7.11). The only condition is that $\sigma_{n}$ in (7.8) is fixed or a bounded function of $\mu$ on $[0, \infty)$. In this event $z-\sigma_{n}$ can be viewed as large parameter in $Q_{n}$, although $z>\sigma_{n}$ is enough.

For the construction of error bounds it is sufficient that $f_{n}(t)$ is continuous on $[0, \infty)$, i.e., that $f$ belongs to the continuity class $C^{2 n}([0, \infty)$ ) (see (5.4)). A different point is that, as remarked earlier, a slight modification is needed when $f_{n}(\mu)=0$. A special case is $\mu=0$, where $f_{n}, \bar{f}_{n}$ vanish for $n \geqq 1$. In that case we can define $\sigma_{n}=0$. When we construct error bounds, the assumption (4.4) on the algebraic growth is only needed for $t \geqq 0$. Another assumption on $f$ may be that $\sigma_{n}$ of (7.8) is a bounded function of $\mu$. A proper choice of $M_{n}$, for instance by making $M_{n}$ a function of $\mu$, will yield a wide class of admissible functions $f$. The construction of error bounds is not enough to investigate the nature of the asymptotic expansion. However, when $\sigma_{n}, M_{n}, f_{n}(\mu) / f(\mu)(f(\mu) \neq 0)$ are bounded functions of $\mu$ on $[0, \infty)$ for each $n \geqq 0$, then we can use the Poincaré-type scale $\left\{z^{-s}\right\}$, and the uniformity with respect to $\mu$ in $[0, \infty)$ easily follows.

A possible approach to compute $M_{n}$ and $\sigma_{n}$ of (7.8) is to start with trial values of $M_{n}$ satisfying (7.2). Then we compute

$$
\sigma_{n}=\sup _{t \geqq 0} \tilde{f}_{n}(t), \quad \mu \text { fixed in }[0, \infty),
$$

where

$$
\tilde{f}_{n}(t)=\frac{\log \left|f_{n}(t) /\left[M_{n} f_{n}(\mu)\right]\right|}{t-\mu-\mu \log (t / \mu)}, \quad t \neq \mu, \quad f_{n}(\mu) \neq 0 .
$$

For two examples we have computed $\sigma$-values. A third example is considered in $\S 9$ for the incomplete beta function.

Example 7.1. $f(t)=1 /(1+t)$. We have

$$
f_{2}(\mu)=\frac{\mu(\mu-2)}{(1+\mu)^{5}}, \quad f_{2}(t)=\frac{t(\mu t-\mu-2)}{(1+\mu)^{3}(1+t)^{3}} .
$$

Since $f_{2}(\mu)$ vanishes at $\mu=2$, we replace it by

$$
f_{2}^{*}(\mu)=\frac{\mu(1+|\mu-2|)}{(1+\mu)^{5}} .
$$

We consider three choices of $M_{2}$ and we obtain $\sigma_{2}$ via (7.12) for several values of $\mu$. We also show corresponding values of $Q_{2}$ of (7.9) for $z=5$. For larger values of $z, Q_{2}$ is closer to unity. The results are shown in Table 7.1. It follows that the remainder $z^{-2} \bar{f}_{2}$ of (3.3) is rather close to the first neglected term $z^{-2} f_{2}(\mu)$ for the values of $\mu$ and $z$ used in the table. Larger values of $\mu$ and $z$ confirm this tendency even better. 
TABLE 7.1

\begin{tabular}{r|c|c|c|c|c|c}
\hline \multirow{2}{*}{$\mu$} & \multicolumn{2}{|c|}{$M_{2}=1.1$} & \multicolumn{2}{c|}{$M_{2}=1.5$} & \multicolumn{2}{c}{$M_{2}=2.0$} \\
\cline { 2 - 7 } & $\sigma_{2}$ & $Q_{2}$ & $\sigma_{2}$ & $Q_{2}$ & $\sigma_{2}$ & $Q_{2}$ \\
\hline 0 & 0 & 1 & 0 & 1 & 0 & 1 \\
1 & -0.64 & 0.9397 & -0.87 & 0.9210 & -0.98 & 0.9120 \\
5 & 0.02 & 1.0023 & -0.01 & 0.9989 & -0.04 & 0.9960 \\
10 & 0.04 & 1.0038 & 0.00 & 1.0004 & -0.01 & 0.9993 \\
25 & 0.11 & 1.0108 & 0.03 & 1.0031 & 0.01 & 1.0014 \\
50 & 0.08 & 1.0079 & 0.02 & 1.0024 & 0.01 & 1.0014 \\
100 & 0.05 & 1.0047 & 0.01 & 1.0014 & 0.00 & 1.0001 \\
\hline
\end{tabular}

Example 7.2. $f(t)=\exp [\mu /(1+t)]$. We use (7.3), (7.7) with $n=0$, which gives a bound (7.8) for $\bar{f}_{0}$ of (3.3), i.e., for $z^{\lambda} F_{\lambda}(z)$ of (2.1). The results are shown in Table 7.2 , again with $z=5$.

TABLE 7.2

\begin{tabular}{r|l|l|l|l|l|l}
\hline \multirow{2}{*}{$\mu$} & \multicolumn{2}{|c|}{$M_{0}=1.1$} & \multicolumn{2}{c|}{$M_{0}=1.5$} & \multicolumn{2}{c}{$M_{0}=2.0$} \\
\cline { 2 - 7 } & $\sigma_{0}$ & $Q_{0}$ & $\sigma_{0}$ & $Q_{0}$ & $\sigma_{0}$ & $Q_{0}$ \\
\hline 0 & 0 & 1 & 0 & 1 & 0 & 1 \\
1 & 0.38 & 1.0417 & 0.03 & 1.0027 & -0.02 & 0.9976 \\
5 & 0.66 & 1.0737 & 0.31 & 1.0329 & 0.26 & 1.0270 \\
10 & 0.46 & 1.0501 & 0.26 & 1.0276 & 0.25 & 1.0259 \\
25 & 0.21 & 1.0221 & 0.21 & 1.0216 & 0.21 & 1.0213 \\
50 & 0.18 & 1.0187 & 0.18 & 1.0185 & 0.18 & 1.0184 \\
100 & 0.16 & 1.0162 & 0.16 & 1.0161 & 0.16 & 1.0161 \\
\hline
\end{tabular}

7.2. Error bounds for (3.2). For the construction of error bounds for the remainder of expansion (3.2), we use as comparison function $w_{\sigma}(t, \alpha)$, with $w_{\sigma}$ defined in (7.7). The comparison function $w_{\sigma}(t, \mu)$ may yield unrealistic bounds, when $\alpha$ and $\mu$ are not of the same size. In Theorem 6.2 we used (6.10) in order to get rid of the factor $z$ in $(3.5),(3.6)$. However, this factor is neutralized by the expression $f_{n}(t)-\bar{f}_{n}$ in the integral (the minus-sign is important here). In the following error analysis this expression will not be replaced by $\left|f_{n}(t)\right|+\left|\bar{f}_{n}\right|$.

We write

$$
g_{n}(t)=f_{n}(t)-\bar{f}_{n}, \quad n=0,1, \cdots .
$$

When $g_{n}(\alpha) \neq 0$, we estimate $g_{n}$ as follows:

$$
\left|g_{n}(t)\right| \leqq M_{n}\left|g_{n}(\alpha)\right| w_{\tau_{n}}(t, \alpha)
$$

where $M_{n}$ satisfies (7.2) and $w$ is defined in (7.7). We consider two cases.

(i) $0 \leqq \alpha \leqq \mu$. Starting point is (3.6); (7.15) has to be considered for $t \in[0, \alpha]$. We obtain

$$
\left|\bar{B}_{n}\right| \leqq z M_{n} \alpha^{\alpha \tau_{n}-\lambda} e^{\alpha\left(z-\tau_{n}\right)}\left|g_{n}(\alpha)\right| \int_{0}^{\alpha} t^{\lambda-\alpha \tau_{n}-1} e^{-\left(z-\tau_{n}\right) t} d t
$$

Integration by parts gives

$$
\left|\bar{B}_{n}\right| \leqq \frac{z M_{n}}{\lambda-\alpha \tau_{n}}\left|g_{n}(\alpha)\right|\left[1+\left(z-\tau_{n}\right) g\left(\alpha, \lambda-\alpha \tau_{n}, z-\tau_{n}\right)\right],
$$


where $g$ is defined in (6.5). In Lemma 6.1 it is supposed that in $g(\alpha, \lambda, z)$ the parameters satisfy $0 \leqq \alpha \leqq \lambda / z$. In the present $g$-function this relation holds as well. The only condition is $z>\tau_{n}$. For a better representation of the above bound for $\bar{B}_{n}$ we write

$$
\frac{z}{\lambda-\alpha \tau_{n}}\left|g_{n}(\alpha)\right|=\frac{\lambda}{\lambda-\alpha \tau_{n}} \frac{\left|g_{n}(\alpha)\right|}{\mu} .
$$

The first factor at the right equals $1 /\left(1-\alpha \tau_{n} / \mu z\right)$ which is $1+\mathcal{O}\left(z^{-1}\right)$ as $z \rightarrow \infty$, uniformly with respect to $\alpha$ and $\mu, 0 \leqq \alpha \leqq \mu$. The second factor $\left|g_{n}(\alpha)\right| / \mu$ is properly defined in the limit $\mu \rightarrow 0$, as follows from a similar argument as used for (5.8). By using Lemma 6.1 we obtain for $z>\tau_{n}$

$$
\left|\bar{B}_{n}\right| \leqq \frac{\lambda M_{n}}{\lambda-\alpha \tau_{n}} \frac{\left|g_{n}(\alpha)\right|}{\mu}\left[1+\frac{\alpha}{\bar{\mu}-\alpha} \min \left\{1, \bar{\zeta} \sqrt{\frac{1}{2} \pi\left(z-\tau_{n}\right)}\right\}\right],
$$

where $\bar{\mu}$ and $\bar{\zeta}$ are defined by

$$
\frac{1}{2} \bar{\zeta}^{2}=\alpha-\bar{\mu}+\bar{\mu} \log (\bar{\mu} / \alpha), \quad \bar{\zeta} \geqq 0, \quad \bar{\mu}=\left(\lambda-\alpha \tau_{n}\right) /\left(z-\tau_{n}\right) .
$$

The conditions $0 \leqq \alpha \leqq \mu$ and $z>\tau_{n}$ imply $0 \leqq \alpha \leqq \bar{\mu}$.

(ii) $0 \leqq \mu \leqq \alpha$. We consider (7.15) for $t \geqq \alpha$. Representation (3.5) gives for $z>\tau_{n}$

$$
\left|\bar{B}_{n}\right| \leqq z M_{n}\left|g_{n}(\alpha)\right| G_{0}\left(\alpha, \lambda-\alpha \tau_{n}, z-\tau_{n}\right) .
$$

Using Lemma 6.2, we obtain

$$
\left|\bar{B}_{n}\right| \leqq \frac{M_{n}\left|g_{n}(\alpha)\right|}{(\alpha-\bar{\mu})\left(1-\tau_{n} / z\right)} \min \left\{1, \bar{\zeta} \sqrt{\frac{1}{2} \pi\left(z-\tau_{n}\right)}\right\} .
$$

When $\lambda-\alpha \tau_{n}<0$ we define $\bar{\zeta}=+\infty$, otherwise it is defined by (7.17), with $0 \leqq \bar{\mu} \leqq \alpha$.

Remark 7.1. The numbers $\bar{\zeta}, M_{n}$ and $\tau_{n}$ in (7.16) and (7.18) need not be the same. When $\alpha \rightarrow \bar{\mu}, \bar{\zeta}$ has to be combined with the factor $1 /(\bar{\mu}-\alpha)$, as explained in Remark 6.1 .

Remark 7.2. We can write $g_{n}(t)$ of (7.14) in the form

$$
g_{n}(t)=\tilde{g}_{n}(t)-\frac{1}{z} \bar{f}_{n+1}, \quad \tilde{g}_{n}(t)=f_{n}(t)-f_{n}(\mu) .
$$

Bounds for $\bar{f}_{n+1}$ follow from (7.8). Contributions owing to $\tilde{g}_{n}(t)$ are as in (7.16), (7.18) with $g_{n}(\alpha)$ replaced by $\tilde{g}_{n}(\alpha)$. Observe that (see (2.7))

$$
\tilde{g}_{n}(\alpha) /(\alpha-\mu)=B_{n}(\alpha),
$$

which shows up in (7.18) when we use (7.19). There is something to recommend about the approach based on (7.19). The point is that $g_{n}$ of (7.14) may be difficult to evaluate without the splitting in (7.19). Furthermore, $M_{n}$ and $\tau_{n}$ of (7.15) may depend on $z$. However, this dependence will be very weak when $z$ is large.

8. A loop integral with analogue asymptotic features. In previous papers [11], [12] we stated the analogy between the following integrals:

$$
\begin{aligned}
& F_{\lambda}(z)=\frac{1}{\Gamma(\lambda)} \int_{0}^{\infty} t^{\lambda-1} e^{-z t} f(t) d t, \\
& G_{\lambda}(z)=\frac{\Gamma(\lambda+1)}{2 \pi i} \int_{-\infty}^{(0+)} t^{-\lambda-1} e^{z t} f(t) d t,
\end{aligned}
$$


where (8.1) is the complete integral given in (2.1). The contour in (8.2) starts and ends at $t=-\infty$ (respectively, with arg $t=-\pi, \arg t=\pi$ ), and encircles the origin in positive direction. The analogy, from an asymptotic point of view, is that of their asymptotic expansions:

$$
\begin{aligned}
& z^{\lambda} F_{\lambda}(z) \sim \sum_{s=0}^{\infty} f_{s}(\mu) z^{-s} \\
& z^{-\lambda} G_{\lambda}(z) \sim \sum_{s=0}^{\infty}(-1)^{s} f_{s}(\mu) z^{-s},
\end{aligned}
$$

as $z \rightarrow \infty$; (8.3) is considered in the present paper, for instance in (2.4). The construction of (8.4) is based on the same integration by parts procedure, by using Hankel's integral for the reciprocal gamma function. That is, (8.2) reduces to $z^{\lambda}$, when $f$ is the identity. Conditions on $f$, especially the domain of holomorphy, have to be modified, before we can state that (8.2) has (8.4) as a uniform expansion with $z$ as the large parameter and $\mu=\lambda / z$ as uniformity parameter.

It seems that the following integral

$$
G_{\lambda}(z, \alpha)=\frac{\Gamma(\lambda+1)}{2 \pi i} \int_{-\infty}^{\left(0^{+}\right)} t^{-\lambda} e^{z t} g(t) \frac{d t}{t-\alpha}
$$

is the relative of $F_{\lambda}(z, \alpha)$ defined in (1.1). That is, (8.5) has four asymptotic phenomena that are in some sense equivalent to the four discussed in $\S 1$ for (1.1). However, the asymptotic expansions show an interesting difference, although the characteristics of both are exactly the same.

8.1. Uniform approximation for loop integrals. We suppose that the contour in (8.5) cuts the positive real axis at the point $t_{0}$. We first give (8.5) for $g=1$. Multiplying by $\exp (-\alpha z)$ and differentiating with respect to $z$, we obtain (8.2) with $f=1$. Integrating with respect to $z$, and taking into account some limiting values, we obtain two forms for (8.5) with $g=1$ :

$$
G_{\lambda}(z, \alpha)=\lambda \alpha^{-\lambda} e^{\alpha z} \begin{cases}\gamma(\lambda, \alpha z), & t_{0}>\alpha, \\ (-1) \Gamma(\lambda, \alpha z), & 0<t_{0}<\alpha,\end{cases}
$$

where $\gamma(a, x), \Gamma(a, x)$ are incomplete gamma functions. The transition from one form to the other in (8.6) also follows from (8.5), by shifting the contour across the pole at $t=\alpha$, and using $\gamma(a, x)+\Gamma(a, x)=\Gamma(a)$.

Suppose now that $0<t_{0}<\alpha$. Writing $g(t)=g(\alpha)+[g(t)-g(\alpha)]$, we obtain for $(8.5)$

$$
G_{\lambda}(z, \alpha)=G_{\lambda}(z)-\lambda g(\alpha) \alpha^{-\lambda} e^{\alpha z} \Gamma(\lambda, \alpha z)
$$

where

$$
\begin{aligned}
& G_{\lambda}(z)=\frac{\Gamma(\lambda+1)}{2 \pi i} \int_{-\infty}^{\left(0^{+}\right)} t^{-\lambda-1} e^{z t} h(t) d t, \\
& h(t)=t \frac{g(t)-g(\alpha)}{t-\alpha} .
\end{aligned}
$$

Therefore, assuming appropriate conditions on $g$, and hence on $h$, the asymptotic expansion of $G_{\lambda}(z)$ is given in (8.4) with $f_{s}(\mu)$ replaced by $h_{s}(\mu)$. The latter are generated as $f_{s}(\mu)$ in $(2.5)$, with $f_{0}$ replaced by $h$ and $f_{s}$ by $h_{s}$. 
We conclude that, apart from normalization, (8.7) has with (8.4) a similar expansion as $F_{\lambda}(z, \alpha)$ in (2.6). An interesting difference is that now the incomplete gamma function does not multiply a full asymptotic expansion but just one term involving $g(\alpha)$. This gives a simpler asymptotic problem. For instance, the construction of error bounds only applies to $G_{\lambda}(z)$, i.e., for the complete integral (8.2), where $f$ is replaced by $h$ of (8.8).

A representation for the remainders in the expansion of $G_{\lambda}(z)$ in (8.7) follows by writing

$$
\begin{aligned}
& z^{-\lambda} G_{\lambda}(z)=\sum_{s=0}^{n-1}(-1)^{s} h_{s}(\mu) z^{-s}+(-1)^{n} z^{-n} \bar{h}_{n}, \\
& \bar{h}_{n}=\frac{z^{-\lambda} \Gamma(\lambda+1)}{2 \pi i} \int_{-\infty}^{\left(0^{+}\right)} t^{-\lambda-1} e^{z t} h_{n}(t) d t,
\end{aligned}
$$

where $h_{n}(t)$ is generated by the recursion (2.5), with starting function $h_{0}=h$ defined in (8.8).

8.2. Error bounds for loop integrals. For the construction of error bounds, we select a special contour in (8.10). Writing

$$
t^{-\lambda} e^{z t}=e^{z\left[\rho e^{i \theta}-\mu \log \rho-i \mu \theta\right]}, \quad t=\rho e^{i \theta},
$$

we see that the imaginary part of the phase function will vanish, when we take

$$
\rho=\rho(\theta)=\mu \theta / \sin \theta, \quad-\pi<\theta<\pi .
$$

This defines the path of steepest descent through the saddle point $t=\mu$. Integrating (8.10) with respect to the parameter $\theta$ along this contour, we use

$$
\frac{1}{t} \frac{d t}{d \theta}=\frac{1}{\rho} \frac{d \rho}{d \theta}+i
$$

giving

$$
\bar{h}_{n}=\frac{\Gamma(\lambda+1) \lambda^{-\lambda} e^{\lambda}}{2 \pi} \int_{-\pi}^{\pi} e^{-\lambda \phi(\theta)} \tilde{h}_{n}(t) d \theta,
$$

where

$$
\begin{aligned}
& t=\rho e^{i \theta}=\mu[\theta \operatorname{cotg} \theta+i \theta], \\
& \tilde{h}_{n}(t)=\frac{1}{i t} \frac{d t}{d \theta} h_{n}(t), \\
& \phi(\theta)=-\theta \operatorname{cotg} \theta+\log \frac{\theta}{\sin \theta}+1 .
\end{aligned}
$$

A bound for $\tilde{h}_{n}$ is obtained by writing as in (7.8)

$$
\left|\tilde{h}_{n}(t)\right| \leqq M_{n}\left|h_{n}(\mu)\right| e^{\mu \delta_{n} \phi(\theta)},
$$

where $M_{n}$ satisfies (7.2), $\delta_{n}<z$, and where it is assumed that $h_{n}(\mu) \neq 0$. We obtain as in (7.9)

$$
\left|\bar{h}_{n}\right| \leqq M_{n} \tilde{Q}_{n}\left|h_{n}(\mu)\right| \text {, }
$$

where $\tilde{Q}_{n}=1 /\left[\left(1-\delta_{n} / z\right) Q_{n}\right]$. We have used that (8.10) reduces to unity when $h_{n}$ equals unity. 
Observe that to compute error bounds for the expansion (8.9), exactly the same comparison function is used as in (7.8); the forms are different owing to the mapping $t \rightarrow \theta$.

Remark 8.1. Representation (8.7) is obtained under the condition $0<t_{0}<\alpha$, where $t_{0}$ is the point where the contour of (8.5) cuts the real positive axis. When (8.5) is presented with $t_{0}>\alpha, G_{\lambda}(z, \alpha)$ has representation (8.7), with $\Gamma(\lambda, \alpha z)$ replaced by $-\gamma(\lambda, \alpha z)$. This complementary relation is of the same kind as for the real integrals described in (2.14), (2.15).

9. The incomplete beta function. We use the incomplete beta function in the notation

$$
I_{x}(p, q)=\frac{1}{B(p, q)} \int_{0}^{x} \tau^{p-1}(1-\tau)^{q-1} d \tau,
$$

where $B(p, q)=\Gamma(p) \Gamma(q) / \Gamma(p+q)$ is the complete beta function. The asymptotic problem is to give an expansion of $I_{x}(p, q)$ with $p$ as large parameter and $x \in[0,1]$ and $\mu=q / p \in[0, \infty)$ as uniformity parameters. We can use

$$
I_{x}(p, q)=1-I_{1-x}(q, p)
$$

to interchange the role of $p$ and $q$. For information on $I_{x}(p, q)$ we refer to [1, p. 944]. In [10] we considered the asymptotic problem for $I_{x}(p, q)$ for more restricted ranges of the parameters. We believe that the expansions of this section are new in the sense that the uniformity domain of $\mu$ or $q$ is the complete interval $[0, \infty)$. Earlier results prescribed $q$ to belong to a compact subset of $(0, \infty)$ (case (ii) of $\S 1$ ), or $p / q$ to a compact subset of $(0, \infty)$ (case (iv)). Extension to complex values of the parameters is possible, but will not be considered here.

To describe the asymptotic features of (9.1) in more detail, we compute the saddle point of $\tau^{p}(1-\tau)^{q}$. It occurs at

$$
\tau_{0}=\frac{p}{p+q} .
$$

When $p+q$ is large, the value $I_{x}(p, q)$ is very small when $x<\tau_{0}$, and it is close to unity when $x>\tau_{0}$. When $\tau_{0}$ is restricted to a compact subset of $(0,1)$, this transition can properly be described by an error function (normal distribution function); when $\tau_{0} \rightarrow 1$ the basic approximant is an incomplete gamma function. We will show that this function can handle the complete uniformity domain for $q$, i.e., $[0, \infty)$. It is essential to transform (9.1) to the standard form (1.1), by means of a rather complicated transformation.

9.1. Transformation to standard form. A first transformation $\tau \rightarrow e^{-\tau}$ gives

$$
I_{x}(p, q)=\frac{1}{B(p, q)} \int_{-\log x}^{\infty}\left(1-e^{-\tau}\right)^{q-1} e^{-p \tau} d \tau .
$$

Comparing this with (1.1), we observe that it has the standard form when $\lambda=q, z=p$ and $f(t)=\left[\left(1-e^{-\tau}\right) / \tau\right]^{q-1}$. However, for several reasons this choice of $f$ will not give a uniform expansion for the $q$-interval $[0, \infty)$. One reason is that large values of $q$ will have much influence on coefficients $f_{s}(\mu), B_{s}(\alpha)$. Observe that for $f$ dependence only on $\mu$ is assumed in $\S 4$, and not on $\lambda$.

A better way for transforming (9.4) in (1.1) is to use the mapping $\tau \rightarrow t(\tau)$ defined by

$$
\tau-\mu \log \left(1-e^{-\tau}\right)=t-\mu \log t+A(\mu),
$$


where $\mu=q / p$. The left-hand side has a saddle point at

$$
\tau_{1}=\log (\mu+1)=-\log \tau_{0},
$$

the right-hand side at $t=\mu$. To make the mapping properly defined we require the correspondences

$$
\tau=0 \leftrightarrow t=0, \quad \tau=\tau_{1} \leftrightarrow t=\mu, \quad \tau=+\infty \leftrightarrow t=+\infty .
$$

The middle one gives

$$
A(\mu)=(1+\mu) \log (1+\mu)-\mu .
$$

The point $\tau=-\log x$, the lower end point of integration in (9.4), is mapped to $t=\alpha$, which is defined by the implicit relation

$$
-\log x-\mu \log (1-x)=\alpha-\mu \log \alpha+A(\mu),
$$

with corresponding points

$$
x=0 \leftrightarrow \alpha=+\infty, \quad x=\tau_{0} \leftrightarrow \alpha=\mu, \quad x=1 \leftrightarrow \alpha=0 .
$$

Observe that the middle one satisfies (9.9) due to the choice (9.8). In fact, the mappings (9.5) and (9.9) are the same, up to parametrization.

The transformed version of (9.4) is

$$
I_{x}(p, q)=\frac{e^{-p A(\mu)}}{B(p, q)} \int_{\alpha}^{\infty} t^{q-1} e^{-p t} f(t) d t,
$$

where

$$
f(t)=\frac{t}{1-e^{-\tau}} \frac{d \tau}{d t}=\frac{t-\mu}{1-(1+\mu) e^{-\tau}} .
$$

The regularity of the transformation (9.5), and that of $f$, is extensively discussed in $[12, \S 4]$. From that analysis it follows that $f$ satisfies the conditions of $\S 4$. In (4.1) we have to take $\kappa=\frac{1}{2}$, and $d_{0}$ and $\delta$ both somewhat less than $\sqrt{2 \pi} . \Omega_{0}$ is a parabolashaped domain, and for the number $p$ in (4.4) we take $p=1$ (which follows easily from (9.12)). The function $f$ is positive on $[0, \infty) ; f(0)=1, f(\mu)=\sqrt{1+\mu}$. We verified numerically that

$$
\sup _{\substack{t \geqq 0 \\ \mu \geqq 0}} \frac{f(t)}{1+t}=1 .
$$

Therefore, $M(\mu)$ of (4.4) will not deviate very much from unity, especially when $\delta$ and $d_{0}$ are small.

9.2. Uniform expansion of incomplete beta function. In the notation of (1.1), (2.1), (2.9), we can write

$$
\begin{aligned}
& I_{x}(p, q)=\frac{e^{-p A(\mu)} \Gamma(p+q)}{\Gamma(p)} F_{q}(p, \alpha), \\
& F_{q}(p, \alpha)=Q(q, \alpha p) F_{q}(p)+\frac{\alpha^{q} e^{-\alpha p}}{p \Gamma(q)} B_{q}(p, \alpha) .
\end{aligned}
$$

However, the "complete" integral $F_{q}(p)$ can be written in this case in terms of known functions. Since $I_{1}(p, q)=1$, we have

$$
F_{q}(p)=e^{p A(\mu)} \Gamma(p) / \Gamma(p+q)=\left(\frac{p+q}{p}\right)^{p+q} e^{-q} \Gamma(p) / \Gamma(p+q)
$$


Although it is possible to give for $F_{q}(p)$ an expansion as in $(2.4)$ (see [12, $\S 4$ for a related expansion), it is more attractive now to write (9.13) in the form

$$
I_{x}(p, q)=Q(q, \alpha p)+\frac{\alpha^{q} e^{-\alpha p+q}}{p B(p, q)}\left(\frac{p}{p+q}\right)^{p+q} B_{q}(p, \alpha) .
$$

By using (9.9), and (2.11) we can write

$$
I_{x}(p, q)=Q(q, \alpha p)+\frac{x^{p}(1-x)^{q}}{p B(p, q)} B_{q}(p, \alpha), \quad B_{q}(p, \alpha) \sim \sum_{s=0}^{\infty} \frac{B_{s}(\alpha)}{p^{s}} \quad \text { as } p \rightarrow \infty .
$$

It follows that we have only to consider the asymptotic expansion for $B_{q}(p, \alpha)$, which, however, is not the simpler one of (2.4), (2.11). The first coefficient is

$$
B_{0}(\alpha)=\frac{f(\alpha)-f(\mu)}{\alpha-\mu}
$$

with

$$
f(\alpha)=\frac{\alpha-\mu}{1-(1+\mu) x}, \quad f(\mu)=\sqrt{1+\mu} .
$$

Special values are

$$
B_{0}(0)=\frac{\sqrt{1+\mu}-1}{\mu}, \quad B_{0}(\mu)=\lim _{\alpha \rightarrow \mu} f^{\prime}(\alpha)=\frac{\mu-1+\sqrt{1+\mu}}{3 \mu}, \quad B_{0}(\infty)=1,
$$

and they satisfy $0 \leqq B_{0}(0) \leqq B_{0}(\mu) \leqq B_{0}(\infty)$, for $\mu \geqq 0$.

In fact, all $B_{s}(\alpha)$ can be expressed in terms of $\alpha, x$ and $\mu$, and those three are related by (9.9). When $\alpha \neq \mu$, an explicit representation of $B_{s}(\alpha)$ in terms of say $x$ and $\mu=q / p$ is not possible, since (9.9) cannot be solved explicitly for $\alpha$. When $\mu=0$, (9.5) reduces to the identity mapping and $f$ of (9.12) becomes $t /[1-\exp (-t)]$. The latter has singularities at $t= \pm 2 \pi i, \pm 4 \pi i, \cdots$. When $\mu>0$, singular points of $f$ originate from these points, of which $\pm 2 \pi i$ are most important. The singular points of $f$ starting from $\pm 2 \pi i(\mu=0)$ are located in the half-plane $\operatorname{Re} t>0$. For large values of $\mu$ they are approximately near $\mu+2 \sqrt{\pi \mu} \exp ( \pm i \pi / 4)$. The value $\kappa=\frac{1}{2}$ in (4.1) comes from $\sqrt{\mu}$ in this asymptotic value. The coefficients $B_{s}(\alpha)$ have the same domain of regularity as $f(\alpha)$. Since $M(\mu)=\mathscr{O}(1), p=1, \kappa=\frac{1}{2}, \bar{\kappa}=0$, we have for (5.7)

$$
B_{s}(\alpha)=\mathcal{O}(1+\mu+\alpha)^{1 / 2}, \quad s=0,1,2, \cdots, \quad \mu \geqq 0, \quad \alpha \geqq 0 .
$$

This estimate gives a good impression of the asymptotic nature of the expansion in (9.16), although the estimate for $s=0$ seems to be too large (cf. (9.18)).

9.3. Uniform expansion based on a loop integral. An interesting variant of (9.16) is obtained by using a contour integral for $I_{x}(p, q)$ in the complex plane, and by applying the method of $\S 8$. Consider the integral

$$
I=\frac{1}{2 \pi i} \int_{c-i \infty}^{c+i \infty} t^{-p}(1-t)^{-q} \frac{d t}{t-x}, \quad 0<c<1,
$$

with $p+q>0$ and $0<x<c$. When $t \in(0,1)$ the phases of $t,(1-t)$ and of the multivalued functions are zero. By deforming the contour around the negative axis, we obtain for $p<1$

$$
\begin{aligned}
I & =x^{-p}(1-x)^{-q}-\frac{\sin \pi p}{\pi} \int_{0}^{\infty} \tau^{-p}(1+\tau)^{-q} \frac{d \tau}{\tau+x} \\
& =x^{-p}(1-x)^{-q} I_{x}(p, q),
\end{aligned}
$$


where we used formulas $6.1 .17,15.3 .1,15.3 .3,15.3 .4,26.5 .23$ and 26.5 .2 of [1]. It follow that

$$
I_{x}(p, q)=\frac{x^{p}(1-x)^{q}}{2 \pi i} \int_{c-i \infty}^{c+i \infty} \tau^{-p}(1-\tau)^{-q} \frac{d \tau}{\tau-x}, \quad 0<c<1,
$$

with $0<x<c$. The restriction $p<1$, which is needed to evaluate the contour integr: for $I$, can be dropped by using the principle of analytic continuation. We still nee the condition $p+q>0$ for convergence at infinity.

Representation (9.20) is the analogue of (9.1). To obtain standard form (8.5), w use the transformation $\tau \rightarrow \exp (-\tau)$. This gives

$$
I_{x}(p, q)=\frac{x^{p}(1-x)^{q}}{2 \pi i} \int_{-\infty}^{\left(0^{+}\right)} e^{p t}\left(1-e^{-\tau}\right)^{q} \frac{d \tau}{1-x e^{\tau}}
$$

which is the analogue of (9.4). The contour cuts the positive real $\tau$-axis at a point such that $\tau_{0}<-\log x$. A final transformation

$$
\tau-\mu \log \left(1-e^{-\tau}\right)=t-\mu \log t+A(\mu),
$$

where $\mu=q / p$ and $A(\mu)$ is given in (9.8), gives

$$
I_{x}(p, q)=x^{p}(1-x)^{q} e^{-q}(1+q / p)^{p+q} \frac{1}{2 \pi i} \int_{-\infty}^{\left(0^{+}\right)} e^{p t} t^{-q} \frac{g(t)}{t-\alpha} d t,
$$

where $\alpha$ is defined in (9.9), and

$$
g(t)=\frac{t-\alpha}{1-x e^{\tau}} \frac{d \tau}{d t}
$$

A relation for $d \tau / d t$ is given in (9.12). The contour in (9.23) cuts the positive $t$-ax at a point $t_{0}$ satisfying $t_{0}<\alpha$. Splitting off the pole, we obtain for (9.23)

$$
I_{x}(p, q)=Q(q, \alpha p)+x^{p}(1-x)^{q} e^{-q}(1+q / p)^{p+q} G_{q}(p) / \Gamma(q+1),
$$

with $G_{q}(p)$ as in (8.8). Here we used $g(\alpha)=-1$, which follows from (9.24) by l'Hôpital rule (observe that $t=\alpha$ corresponds with $\tau=-\log x$ in (9.22)).

Observe that the transformations (9.5) and (9.22) are exactly the same. The rea corresponding points in (9.7) determine the mapping in the complex plane. Therefor no new correspondences have to be defined for (9.22). In fact, the mapping has a ver global character. As remarked earlier, we have investigated the mapping (9.5) in [12. with emphasis on what is happening in a neighbourhood of $\mathbb{R}^{+}$. However, the domai of regularity extends to the full half-plane $\operatorname{Re} t \leqq 0$. To understand the mapping in th complex plane, it is instructive to see that the contours of steepest descent in (9.21) (9.23) are mapped onto each other. On these contours the imaginary parts in the left and right-hand side of (9.22) vanish.

Comparing (9.15) and (9.25), we conclude that the function $B_{q}(p, \alpha)$ has (in th special case of this section) an asymptotic expansion which corresponds to that of "complete" integral (8.2). We have

$$
B_{q}(p, \alpha)=\frac{\Gamma(p+1) e^{-q}(1+q / p)^{p+q}}{q \Gamma(p+q)} G_{q}(p),
$$

where $G_{q}(p)$ is given in (8.8), with expansion as in (8.9); $g$ is defined in (9.24).

By using (7.10) and (8.9) we can write (9.26) in the form

$$
B_{q}(p, \alpha)=\frac{\sqrt{\mu+1}}{\mu} \frac{\Gamma^{*}(p)}{\Gamma^{*}(p+q)} p^{-q} G_{q}(p), \quad p^{-q} G_{q}(p) \sim \sum_{s=0}^{\infty}(-1)^{s} h_{s}(\mu) p^{-s} \text {. }
$$


Therefore, the approach based on a complex contour gives for $I_{x}(p, q)$ a simpler asymptotic expansion and simpler error bounds than the approach based on, say, (9.11). The computation of the coefficients $h_{s}(\mu)$ is not a simple problem. Also, the bound for $\left|h_{n}(t)\right|$ in (8.13) has to be computed on a contour in the complex plane. But the form of the error bound (8.14) is much simpler than those obtained in $\S 7$.

To show some of the steps needed to evaluate the coefficients $h_{s}(\mu)$ in (9.27), we compute $h_{0}(\mu)$ and its limiting form for $\alpha \rightarrow \mu$. We have, using $h$ of (8.8) and $g$ of (9.24),

$$
g(\mu)=\frac{\mu-\alpha}{1-(1+\mu) x} \frac{d \tau}{d t}
$$

where the derivative is evaluated at $t=\mu$. From (9.12), we have

$$
\lim _{t \rightarrow \mu} \frac{d \tau}{d t}=\frac{1}{\mu+1} \lim _{t \rightarrow \mu} \frac{t-\mu}{1-(1+\mu) e^{-\tau}}=\frac{1}{\mu+1} \frac{d t}{d \tau}
$$

by using l'Hôpital's rule. Hence

$$
\frac{d \tau}{d t}=\frac{1}{\sqrt{\mu+1}} \text { at } t=\mu .
$$

The square root has a + sign, since $\tau$ is an increasing function of $t$ on $[0, \infty)$. Earlier we computed $g(\alpha)=-1$. So we have

$$
h_{0}(\mu)=\frac{\mu}{\mu-\alpha}\left[\frac{(\mu-\alpha) / \sqrt{\mu+1}}{1-(\mu+1) x}+1\right], \quad \mu \neq \alpha .
$$

To evaluate this at $\alpha=\mu$, we have to investigate the relation between $x$ and $\alpha$ in more detail. From (9.9) it follows that

$$
\alpha[(\mu+1) x-1] \frac{d x}{d \alpha}=x(1-x)(\alpha-\mu) .
$$

Substituting the expansion

$$
x=\frac{1}{\mu+1}+c_{1}(\alpha-\mu)+c_{2}(\alpha-\mu)^{2}+\cdots,
$$

we obtain

$$
c_{1}=-(1+\mu)^{-3 / 2}, \quad c_{1} c_{2}=\frac{1}{3 \mu(1+\mu)^{3}}\left[\frac{1-\mu}{\sqrt{1+\mu}}-1\right] .
$$

When $\alpha \rightarrow \mu, h_{0}(\mu)$ of (9.28) has the expansion

$$
h_{0}(\mu)=-\mu c_{2} / c_{1}+\mathcal{O}(\mu-\alpha) \text {. }
$$

Hence

$$
\lim _{\alpha \rightarrow \mu} h_{0}(\mu)=\frac{1}{3}\left[1-\frac{1-\mu}{\sqrt{\mu+1}}\right] .
$$

It follows that $B_{0}(\alpha)$ of (9.17) and $h_{0}(\mu)$ of (9.29) are related by

$$
B_{0}(\alpha)=\frac{\sqrt{\mu+1}}{\mu} h_{0}(\mu) \text {. }
$$


A relation between the higher coefficients is obtained as follows. Comparing (9.16) with (9.27), we arrive at the formal identity

$$
\sum_{s=0}^{\infty} B_{s}(\alpha) p^{-s}=\frac{\sqrt{\mu+1}}{\mu} \frac{\Gamma^{*}(p)}{\Gamma^{*}(p+q)} \sum_{s=0}^{\infty}(-1)^{s} h_{s}(\mu) p^{-s} .
$$

We can expand

$$
\frac{\Gamma^{*}(p)}{\Gamma^{*}(p+q)} \sim \sum_{s=0}^{\infty} d_{s}(\mu) p^{-s}, \quad d_{0}(\mu)=1
$$

by dividing the two expansions for $\Gamma^{*}(p)$ and $\Gamma^{*}(p(1+\mu))$ (see also $[12, \S 4]$ ). Thus we obtain

$$
B_{s}(\alpha)=\frac{\sqrt{\mu+1}}{\mu} \sum_{r=0}^{s}(-1)^{r} h_{r}(\mu) d_{s-r}(\mu),
$$

which is (9.30) for $s=0$.

9.4. Some numerical results. We used the method of $\S 7.2$ to compute an upper bound for $B_{q}(p, \alpha)$. That is, we computed $M_{0}$ and $\tau_{0}$ for (7.16), (7.18), and we evaluated the expression at the right-hand sides of these inequalities. For $z=p=10$ (10) 100 these expressions were evaluated at the $x$, $\mu$-grid with $x=0.05(0.05) 0.95, \mu=1$ (1) 10. For each $z$ the maximal value occurred at $x=0.05, \mu=1$. The corresponding $\alpha$-value for this $x, \mu$-pair is $4.06254 \cdots$. The upper bounds show an interesting feature. For $z=10$ it is 0.97111 and it steadily increases to 0.97371 for $z=100$. The ratio of this last upper bound and $B_{0}(\alpha)$ of $(9.17)(=0.64933 \cdots)$ equals $1.500 \cdots$, which is the number $M_{0}$ that we used in (7.15), (7.16) and (7.18). We observe that the computed upper bounds are slightly less than $M_{0} B_{0}(\alpha)$, and tend to this value when $z$ increases. The choice $M_{0}=1.1$ showed the same features: the upper bound of $B_{q}(p, \alpha)$ is slightly less than $M_{0} B_{0}(\alpha)$.

The numerical experiments yield the following conclusion:

$$
\sup B_{q}(p, \alpha)=1, \quad p \text { fixed, }
$$

where the supremum is taken over $x \in[0,1]$ (or $\alpha \geqq 0$ ) and $\mu=q / p \in[0, \infty$ ). The maximum is assumed at $x=0(\alpha=\infty)$ and $\mu=0$. See also (5.11); for $n=0$ this limit $L$ equals 1 . Incidentally, (5.9) gives for $n=0$

$$
B_{q}(p, \alpha)=p[\log p-\psi(p)]
$$

at $\alpha=0, \mu=0$, where $\psi(p)$ is the logarithmic derivative of the gamma function. This follows from well-known representations of this function, and from the fact that $f$ of (9.12) equals $t /(1-\exp (-t))$ at $\mu=0$. From (9.13) and the asymptotic expansion of the $\psi$-function, it follows that

$$
B_{0}(p, 0)=\frac{1}{2}+\mathcal{O}\left(p^{-1}\right) \text { as } p \rightarrow \infty \text {. }
$$

We conjecture that $\frac{1}{2} \leqq B_{q}(p, \alpha) \leqq 1$ for $\alpha \in[0, \infty)$ (or $\left.x \in[0,1]\right)$, and $\mu=q / p \in[0, \infty)$, and for all $p$ sufficiently large (say $p \geqq 10$ ).

\section{REFERENCES}

[1] M. A. Abramowitz And I. A. Stegun, Handbook of mathematical functions, National Bureau of Standards Applied Mathematics Series 55, Washington D.C., 1964.

[2] N. BLEISTEIN, Uniform asymptotic expansion of integrals with stationary point near algebraic singularity, Comm. Pure Appl. Math., 19 (1970), pp. 353-370. 
[3] A. ERDÉLyI AND M. WyMAN, The asymptotic evaluation of certain integrals, Arch. Rational Mech. Anal., 14 (1963), pp. 217-260.

[4] A. ERDÉLYI, Asymptotic evaluation of integrals involving a fractional derivative, this Journal, 14 (1974), pp. 159-171.

[5] W. GAUTSCHI, A computational procedure for the incomplete gamma functions, ACM Trans. Math. Software, 5 (1979), pp. 466-481.

[6] F. W. J. Olver, Asymptotics and Special Functions, Academic Press, New York, 1974.

[7] Unsolved problems in the asymptotic estimation of special functions, in Theory and Application of Special Functions, R. Askey, ed., Academic Press, New York, 1975, pp. 99-142.

[8] K. SonI, A note on uniform asymptotic expansions of incomplete Laplace integrals, this Journal, 14 (1983), pp. 1015-1018.

[9] N. M. TEMME, Remarks on a paper of A. Erdélyi, this Journal, 7 (1976), pp. 767-770.

, The uniform asymptotic expansion of a class of integrals related to cumulative distribution functions, this Journal, 13 (1982), pp. 239-253.

[11] - Uniform asymptotic expansions of Laplace integrals, Analysis, 3 (1983), pp. 221-249.

[12] Laplace type integrals: transformation to standard form and uniform asymptotic expansions, Quart. Appl. Math., 43 (1985), pp. 103-123.

[13] R. WONG, On uniform asymptotic expansion of definite integrals, J. Approx. Theory, 7 (1973), pp. 76-86.

[14] A. S. ZIL'BERGLEIT, Uniform asymptotic expansions of some definite integrals, U.S.S.R. Comput. Math. and Math. Phys., 16 (1977), pp. 36-44. 